\title{
Ruthenium Anticancer Compounds: Challenges and Expectations
}

\author{
Ioannis Bratsos ${ }^{\mathrm{a}}$, Stephanie Jedner ${ }^{\mathrm{a}}$, Teresa Gianferrara ${ }^{\mathrm{b}}$, and Enzo Alessio*a
}

\begin{abstract}
Two ruthenium compounds, namely [ImH]trans-[RuCl$(\mathrm{Im})(\mathrm{dmso}-\mathrm{S})](\mathrm{NAMI}-\mathrm{A}, \mathrm{Im}=$ imidazole) and $[\mathrm{IndH}]$ trans-[RuCl$\left.{ }_{4}(\mathrm{Ind})_{2}\right](\mathrm{KP} 1019$, Ind = indazole) have already completed phase I clinical trials as anticancer agents. They both have properties different from platinum anticancer drugs: for example, NAMI-A is selectively active against metastases of solid tumors. They show that in the field of anticancer metal drugs a new approach, based on targeted therapies, is possible. After a concise history of ruthenium anticancer compounds, this contribution will focus on ruthenium-dmso complexes and, in particular, on NAMI-A. Particular emphasis is given on the challenges that are inherent to this field: how to develop new anticancer ruthenium compounds and how to select new active compounds that manifest their anticancer activity through non-conventional mechanisms.
\end{abstract}

Keywords: Anticancer - Antimetastatic · Dimethylsulfoxide · Medicinal inorganic chemistry · Ruthenium

\section{Background}

Platinum-based drugs have been in clinical use for cancer therapy for $c a .30$ years. ${ }^{[1]}$ The parent compound of this class of anticancer agents, cis-[ $\left.\mathrm{PtCl}_{2}\left(\mathrm{NH}_{3}\right)_{2}\right]$ (cisplatin), is highly effective in the treatment of testicular and ovarian cancers and is also in use for treating bladder, cervical, head and neck, esophageal, and small cell lung cancer. ${ }^{[2]}$ Over the years cisplatin has been followed by only a handful of clinically approved Pt(II) complexes, the most relevant being carboplatin and oxaliplatin. The second-generation drug carboplatin, $[\mathrm{Pt}(\mathrm{cbdc})$

\footnotetext{
${ }^{\star}$ Correspondence: Prof. Dr. E. Alessio ${ }^{a}$

Tel.: +39040 5583961

Fax: +39040 5583903

E-Mail: alessi@units.it

aDipartimento di Scienze Chimiche

Università di Trieste

Via L. Giorgieri 1

34127 Trieste, Italy

bDipartimento di Scienze Farmaceutiche

Università di Trieste

Piazzale Europa 1

34127 Trieste, Italy
}

$\left.\left(\mathrm{NH}_{3}\right)_{2}\right]($ cbdc $=1$,1-cyclobutane dicarboxylate), is effective against the same type of tumors that respond to cisplatin, but can be administered at significantly higher doses because of its lower toxicity. ${ }^{[1]}$ Remarkably instead oxaliplatin, [Pt(ox)(dach)] (dach $=R, R$-1,2-diaminocyclohexane, $\mathrm{ox}=\mathrm{ox}$ alate), a third-generation platinum drug, is used for treatment of metastatic colorectal cancer.

The square-planar, inert Pt(II) drugs are activated by slow hydrolysis of the anionic ligand(s) (thus they should be defined as prodrugs) and the antitumor properties of the metabolites are associated to their capability of binding genomic DNA in the cell nucleus forming stable intrastrand crosslinks which then block replication and/or prevent transcription.

\section{Classical Chemotherapy and Screening of New Compounds}

Classical anticancer drugs, both inorganic such as cisplatin and organic such as 5-fluorouracil, interfere with the most fundamental aspect of cancer cells, i.e. their rapidly dividing nature. Having DNA as the primary target such drugs are not selective and, as a consequence, they show heavy toxicity towards the host. Since cancer cells replicate their DNA more frequently than normal cells, and are therefore more susceptible to DNA damage, the aim of classical chemotherapy is that of damaging replication and mitotic processes more in tumor cells than in normal cells. Thus clas- sical anticancer agents are typically highly cytotoxic also in in vitro experiments.

In recent years a better understanding of cancer cell behavior became available. In principle, this knowledge should open the way to targeted therapies, i.e. therapies that selectively address and disrupt specific cellular signaling pathways on which the cancer cells rely for growth, proliferation, metastasis formation and angiogenesis (e.g. growth factors and receptors that are upregulated in cancer cells). Targeted therapies are therefore anticipated to be much more selective, even though of less general application, than the classical approach, and are expected to have a lower general toxicity. ${ }^{[3,4]}$

Another point that needs to be stressed concerns the real target of chemotherapy. In fact, metastases of solid tumors, rather than primary tumors themselves, represent the leading cause for cancer death, i.e. metastases are more decisive for tumor prognosis than primary lesions. The surgical removal of primary tumors is now a highly successful procedure. Metastases, because of their multiple locations, low accessibility to surgery and/or radiotherapy, need to be addressed by chemotherapy. However they generally show poor responsiveness to chemotherapy. As a consequence, the need for drugs that are highly effective against metastasis tumors is very important. ${ }^{[5]}$ It should be kept in mind that the cell population in the primary tumor is heterogeneous and only some cell subtypes are malignant, i.e. possess metastatic ability. Metastatic and non-metastatic cancer cells have different gene expression profiles. 
The success of Pt drugs as anticancer agents has stimulated the search for other active transition-metal complexes in cancer chemotherapy. ${ }^{[6]}$ The aim of developing non-platinum anticancer complexes is that of overcoming the main limits of platinum drugs: narrow range of activity (they are scarcely active against a number of malignancies with high social incidence), acquired resistance after treatment, and severe toxicity.

Complexes of transition metals other than platinum may, in principle, exhibit anticancer activity and toxic side-effects markedly different from that of Pt drugs for a number of obvious reasons: they are expected to have different chemical behavior, hydrolytic rates, and mechanism(s) of action. Among the several metals that are currently being investigated for their anticancer activity, ruthenium occupies a prominent position. ${ }^{[3-5,7,8]}$

\section{Chemical Features of Ruthenium Compounds}

Some of the main chemical features of $\mathrm{Ru}$ compounds that might be relevant for their biological activity are listed below. Two main oxidation states are accessible for ruthenium species in physiological solution: $\mathrm{Ru}(\mathrm{II})$ ( $d^{6}$, diamagnetic) and $\mathrm{Ru}(\mathrm{III})$ ( $d^{5}$, paramagnetic). ${ }^{[9]}$ In both oxidation states the $\mathrm{Ru}$ ion is six-coordinate with octahedral geometry (while Pt(II) is squareplanar) and, like $\mathrm{Pt}(\mathrm{II})$, has good affinity for nitrogen and sulfur ligands. Ru(II) species will remain unoxidized in air only if good $\pi$-acceptor ligands are present. Broadly speaking, $\mathrm{Ru}$ complexes have kinetics for ligand exchange similar to those of $\mathrm{Pt}(\mathrm{II})$ species. It is also generally accepted that $\mathrm{Ru}(\mathrm{II})$ complexes are less inert compared to the corresponding Ru(III) species. Finally, the ability of ruthenium to mimic iron in binding to many biological molecules, including serum proteins (e.g. transferrin and albumin) is believed to contribute to the general low toxicity of ruthenium drugs.

In the assumption that active $\mathrm{Ru}$ compounds have to coordinate to some biological target in order to exert their anticancer activity, they are to be considered, like Pt drugs, as prodrugs and must undergo some kind of activation process that eventually leads to metabolites that are the real active species. Activation ultimately will require hydrolysis of some ligands, in order to open up coordination positions for allowing coordination to the target. In order to explain the activity of some very inert $\mathrm{Ru}$ (III) chloro-ammine compounds (such as fac- $\left[\mathrm{RuCl}_{3}\left(\mathrm{NH}_{3}\right)_{3}\right]$ and cis- $\left[\mathrm{RuCl}_{2}\left(\mathrm{NH}_{3}\right)_{4}\right]$ $\mathrm{Cl})$, Clarke and co-workers suggested that hydrolysis might need to be preceded by in vivo reduction (activation by reduction hy- pothesis). ${ }^{[7]}$ This activation pathway might offer some kind of selectivity against solid tumors which are more hypoxic, because of insufficient vascularization, and thus have more reducing environments compared to normal tissues and should facilitate the reduction of $\mathrm{Ru}(\mathrm{III})$ to $\mathrm{Ru}(\mathrm{II})$. Another source of tumor selectively for $\mathrm{Ru}$ compounds might derive from their capability to bind to transferrin. Tumor cells often over-express transferrin receptors compared to normal tissues because of their higher iron requirement and a transferrin-mediated uptake has been evidenced for some Ru species.

\section{Short History of Ruthenium Anticancer Compounds}

Historically, the first ruthenium complexes to be investigated for their anticancer activity were chloro-ammine-Ru(III) compounds that can be thought as $\mathrm{Ru}$ analogs of chloro-ammine-Pt compounds. Clarke and co-workers in 1980 reported anticancer activity for $f a c$ - $\left[\mathrm{RuCl}_{3}\left(\mathrm{NH}_{3}\right)_{3}\right]$ in murine models (Fig.). This compound was not pursued much further since its poor solubility precludes it from adequate formulation as a drug. [7]

In 1984 Mestroni and co-workers studied the anticancer activity of a well-known ruthenium(II) complex, cis-[ $\mathrm{RuCl}_{2}\left(\mathrm{dmso}_{4}\right]$ (1, dmso = dimethyl sulfoxide, Fig.), in comparison with that of cisplatin. ${ }^{[10]} \mathrm{Com}-$ pound 1 was found to be non cytotoxic in vitro and to have an in vivo toxicity at least three orders of magnitude lower than that of cisplatin. Nevertheless, when administered at the maximum tolerated dose, $\mathbf{1}$ showed activity against both the primary tumor and the metastases in mice bearing solid metastasizing tumors. In certain cases its activity was comparable to that of cisplatin with the same treatment schedule, but the $\mathrm{Ru}$ complex had an overall less pronounced host toxicity. These results prompted us to undertake a systematic investigation of the antitumor properties of Ru-dmso complexes. ${ }^{[11]}$ In 1988 the Ru(II) complex trans$\left[\mathrm{RuCl}_{2}\left(\mathrm{dmso}_{4}\right]\right.$ (2, Fig.) was shown to be more active than the cis isomer 1 against Lewis lung carcinoma, a metastasizing murine tumor. On a molar basis compound 2 is $c a$. 20 -fold more toxic than the cis isomer and it is more effective in reducing the number and weight of spontaneous metastases derived from the primary tumor. In experiments in which the primary tumor was removed by surgery, i.e. experiments that closely reflect a clinical event, compound 2 was more effective than cisplatin in prolonging the postsurgical survival time of treated mice. In other words, even though cisplatin was more active in reducing the primary tumor, trans- $\left[\mathrm{RuCl}_{2}(\mathrm{dmso})_{4}\right]$ demonstrated a more selective activity against metastases. ${ }^{[10]}$ This feature, that clearly emerged here for the first time, was later found also for other $\mathrm{Ru}$-dmso complexes (see below).

The 80s saw the first real breakthrough in this field: the group of Keppler demonstrated that two isostructural $\mathrm{Ru}$ (III) complexes $[\mathrm{ImH}]$ trans- $\left[\mathrm{RuCl}_{4}(\mathrm{Im})_{2}\right](\mathrm{ICR}, \mathrm{Im}=$ imidazole) and [IndH] trans-[ $\left.\left[\mathrm{RuCl}_{4}(\mathrm{Ind})_{2}\right)\right]$ (KP1019, Ind = indazole, Fig.) were ac-

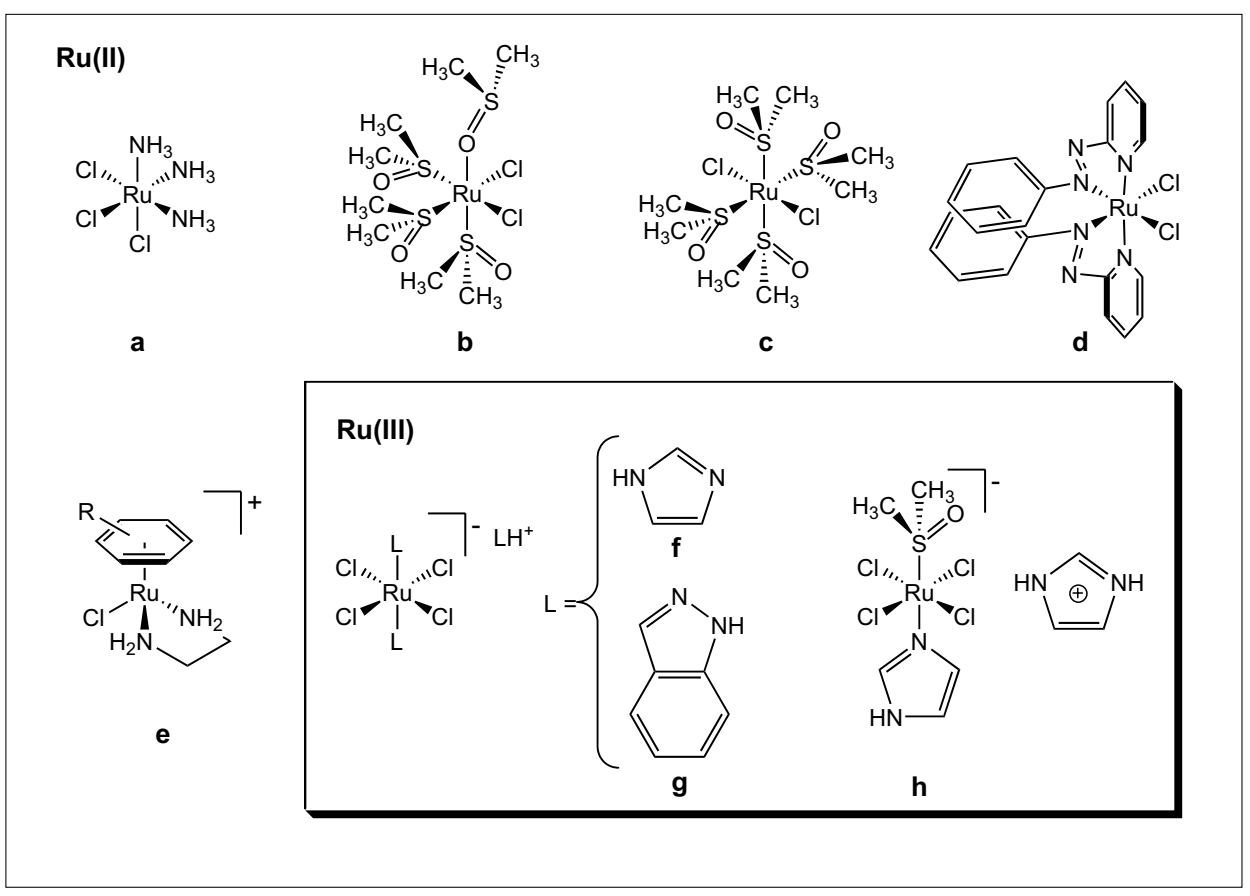

Fig. Structures of some representative $\mathrm{Ru}(\mathrm{II})$ and $\mathrm{Ru}(\mathrm{III})$ (inset) complexes that exhibit anticancer activity: a) fac- $\left[\mathrm{RuCl}_{3}\left(\mathrm{NH}_{3}\right)_{3}\right]$; b) cis- $\left[\mathrm{RuCl}_{2}(\mathrm{dmso})_{4}\right]$ (1); c) trans- $\left[\mathrm{RuCl}_{2}(\mathrm{dmso})_{4}\right](2)$; d) cis- $\left[\operatorname{RuCl}_{2}(\mathrm{azpy})_{2}\right]$; e) the organometallic compounds of general formula $\left[\left(\eta^{6}\right.\right.$-arene) $\left.\mathrm{Ru}(\mathrm{en}) \mathrm{Cl}\right]\left[\mathrm{PF}_{6}\right]$; f) $[\mathrm{ImH}]$ trans- $\left[\mathrm{RuCl}_{4}(\mathrm{Im})_{2}\right]$ (ICR); g) [IndH] trans-[RuCl $\left.\left.{ }_{4}(\mathrm{Ind})_{2}\right](\mathrm{KP} 1019) ; \mathrm{h}\right)[\mathrm{ImH}]$ trans-[RuCl $\left._{4}(\mathrm{Im})(\mathrm{dmso}-\mathrm{S})\right]$ (NAMI-A) 
tive against a number of tumor models and, in particular, showed excellent activity against platinum-resistant colorectal autochthonous tumors.[12] These results clearly supported the basic premise of this field, i.e. that non-platinum active complexes can be effective against platinumresistant tumors, and fostered further research. In that same period, while working on the preparation of the $\mathrm{Ru}$ (II) isomers 1 and $\mathbf{2}$, we isolated and characterized the dmso analogue of Keppler's compounds, $\left[(\mathrm{dmso})_{2} \mathrm{H}\right]$ trans $-\left[\mathrm{RuCl}_{4}(\mathrm{dmso}-\mathrm{S})_{2}\right]$. This compound, which is unsuited for biological testing because of its very rapid hydrolysis at physiological $\mathrm{pH}$, proved to be an excellent precursor for Ru(III) compounds bearing both dmso and heterocyclic N-ligands (L). In the early 90 s we reported that [Na]trans-[ $\left.\mathrm{RuCl}_{4}(\mathrm{Im})(\mathrm{dmso}-\mathrm{S})\right]$ (NAMI), which is structurally similar to ICR with an S-bonded dmso that formally replaces an imidazole, is specifically active against solid metastasizing tumors in mice. ${ }^{[13]}$ At a later stage, NAMI was replaced in pre-clinical experimentations by its imidazolium salt, $[\mathrm{ImH}]$ trans- $\left[\mathrm{RuCl}_{4}(\mathrm{Im})(\mathrm{dmso}-\mathrm{S})\right]$, called NAMI-A (Fig.), which is endowed with the same antimetastatic properties but is a more stable and reproducible solid and consequently is more suited for further development in clinical phase. NAMI-A is capable of effectively inhibiting the development and growth of pulmonary metastases in all experimental models of solid tumors tested in vivo, including the nonsmall cell lung cancer human tumor xenografted in the nude mouse. Conversely, both its activity towards the primary tumor and its in vitro cytotoxicity are negligible when compared to those of cisplatin (for example, cisplatin is cytotoxic against cell cultures at $\mu \mathrm{M}$ concentrations, while NAMI-A is devoid of cytotoxicity up to $\mathrm{mM}$ concentrations).

After extensive preclinical tests, in 1999 NAMI-A began phase I clinical trials at the Netherlands Cancer Institute of Amsterdam; the first compound of ruthenium ever to reach this stage. NAMI-A passed this evaluation after testing in 24 patients showing good tolerability over a wide range of sub-toxic doses without any unexpected toxicity. Although no formal common toxicity criteria (CTC) were developed, painful blister formation was considered dose limiting and the advised dose for further testing of NAMI-A was determined to be 300 $\mathrm{mg} / \mathrm{m}^{2} /$ day. ${ }^{[14]}$ NAMI-A is currently beginning a phase 1-2 clinical trial in therapeutic combination with an anticancer agent mainly active on the primary tumor.

In 2003 also KP1019, the compound developed by Keppler and co-workers, entered phase I clinical trials. ${ }^{[15]}$ This compound, similarly to NAMI-A, is only moderately cytotoxic in conventional cell culture tests.
In more recent years, entirely new classes of $\mathrm{Ru}(\mathrm{II})$ compounds were developed. Reedijk and co-workers reported that complexes with bidentate heterocyclic $\mathrm{N}$-ligands such as cis-[ $\mathrm{RuCl}_{2}$ (azpy) $\left.{ }_{2}\right]$ (azpy = 2-phenylazopyridine, Fig.) possess significant in vitro cytotoxicity. ${ }^{[16]}$ Organometallic Ru(II)-arene compounds, developed by the groups of Sadler ${ }^{[6 \mathrm{~d}, 8]}$ and Dyson, ${ }^{[17]}$ were found to have in vivo activity. In particular the compounds of general formula $\left[\left(\eta^{6}\right.\right.$-arene $\left.) \mathrm{Ru}(\mathrm{en}) \mathrm{Cl}\right]\left[\mathrm{PF}_{6}\right]$ (en = ethylenediamine) developed by Sadler and co-workers (Fig.) showed promising anticancer activity, both in vitro against human cancer cell lines, including the cisplatinresistant A2780cis cell line, and in vivo against animal tumor models. Both classes of compounds are reviewed in specific contributions in this special issue.

\section{NAMI-A}

NAMI-A is endowed with a mechanism of action that, even though still largely unknown, is unrelated to direct tumor cell cytotoxicity (see above) and is thus responsible for the reduced host toxicity of this drug. In other words, even though NAMI-A is capable of interacting with DNA in cellfree medium (like almost any late-transition metal compound that is not completely inert), nuclear DNA is not the primary target of this compound in vivo. Thus, the mechanism of action of this $\mathrm{Ru}(\mathrm{III})$ drug is profoundly distinct from that of platinum drugs. NAMI-A shows several features that might be relevant for its in vivo metastases inhibition, including:

i) inhibition of tumor cell invasion and of matrix metallo proteinases;

ii) upregulation of adhesion and downregulation of angiogenic activity;

iii) ERK1/2 inhibition and caspase activation;

iv) strong interaction with proteins, including albumin, transferrin and integrins.

Several recent publications treat in detail these aspects which will not be discussed further here. ${ }^{[5,13]}$

It is remarkable that the structurally similar NAMI-A and ICR have quite different biological activities. For example ICR, which was found to be active against colorectal tumors, is completely lacking in antimetastatic activity and also its effects on other biological parameters that are typical of NAMI-A (e.g. invasion and adhesion of tumor cells) are much less pronounced. Indeed, the structural similarity between ICR and NAMI-A is deceptive, since the two have remarkably different chemical properties and behaviors in aqueous solution. The main reason for these differences is the dmso ligand that, when bound through sulfur, is a fairly good $\pi$-acceptor and stabilizes Ru(II). As a consequence, the reduction potential of NAMI-A is dramatically more positive than that of ICR $(+235 \mathrm{mV}$ and $-275 \mathrm{mV}$ $v s$ NHE, respectively). ${ }^{[18]}$

\section{Chemical Behavior and Activation of NAMI-A}

The presence of dmso in the coordination sphere of NAMI-A also influences its chemical behavior in aqueous solution, where it is involved in a series of hydrolytic processes whose nature and rate are strongly $\mathrm{pH}$-dependent (Scheme 1). ${ }^{[13]}$ At $37.0^{\circ} \mathrm{C}$ and physiological pH 7.4 (phosphate buffer), the complex is definitely not inert, as it undergoes stepwise hydrolysis of two chlorides within a few minutes. The first hydrolytic step is catalyzed by ruthenium(II) species and its rate is in fact considerably enhanced by the addition of traces of biological reductants, such as ascorbic acid or cysteine. The second hydrolytic process is accompanied by the partial dissociation of the dmso. For comparison, under the same conditions, ICR is remarkably more inert. In vitro, hydrolysis of NAMI-A eventually leads to formation of polymeric oxo- or hydroxo-bridged species characterized by dark color, albeit in vivo this phenomenon is less likely to occur due to the excess of bio-ligands that effectively compete with $\mathrm{OH}^{-}$for coordination. For instance, presence of albumin precludes formation of such species in vitro. ${ }^{[13]}$

On the contrary, NAMI-A is remarkably inert at room temperature and slightly acidic $\mathrm{pH}$ (conditions in which it is administered by infusion): chloride hydrolysis is suppressed and only slow dissociation of dmso occurs.

Most importantly, at physiological $\mathrm{pH}$ stoichiometric amounts of common biological reducing agents (e.g. ascorbic acid, cysteine or glutathione) rapidly and quantitatively reduce NAMI-A to the corresponding dianionic $\mathrm{Ru}(\mathrm{II})$ species trans$\left[\mathrm{RuCl}_{4}(\mathrm{Im})(\mathrm{dmso}-\mathrm{S})\right]^{2-}$, NAMI-AR, which then undergoes rapid stepwise hydrolysis of two chloride ligands (Scheme 1). No loss of dmso or imidazole from the coordination sphere of NAMI-AR, or reoxidation by atmospheric oxygen was observed. Metabolites of NAMI-A that still bear the dmso-S are anticipated to have reduction potentials similar to that of the parent compound and thus to be easily reducible. Remarkably, $\mathrm{Ru}(\mathrm{III})$ and $\mathrm{Ru}$ (II) species with the same net charge, i.e. NAMI-A and mer- $\left[\mathrm{RuCl}_{3}\left(\mathrm{H}_{2} \mathrm{O}\right)\right.$ $(\operatorname{Im})($ dmso-S $)]^{-}$have comparable rates for chloride dissociation.

For comparison, the redox behavior of ICR is much more controversial. Osella and co-workers reported that ICR (as sodium salt) is reduced in vitro within min- 


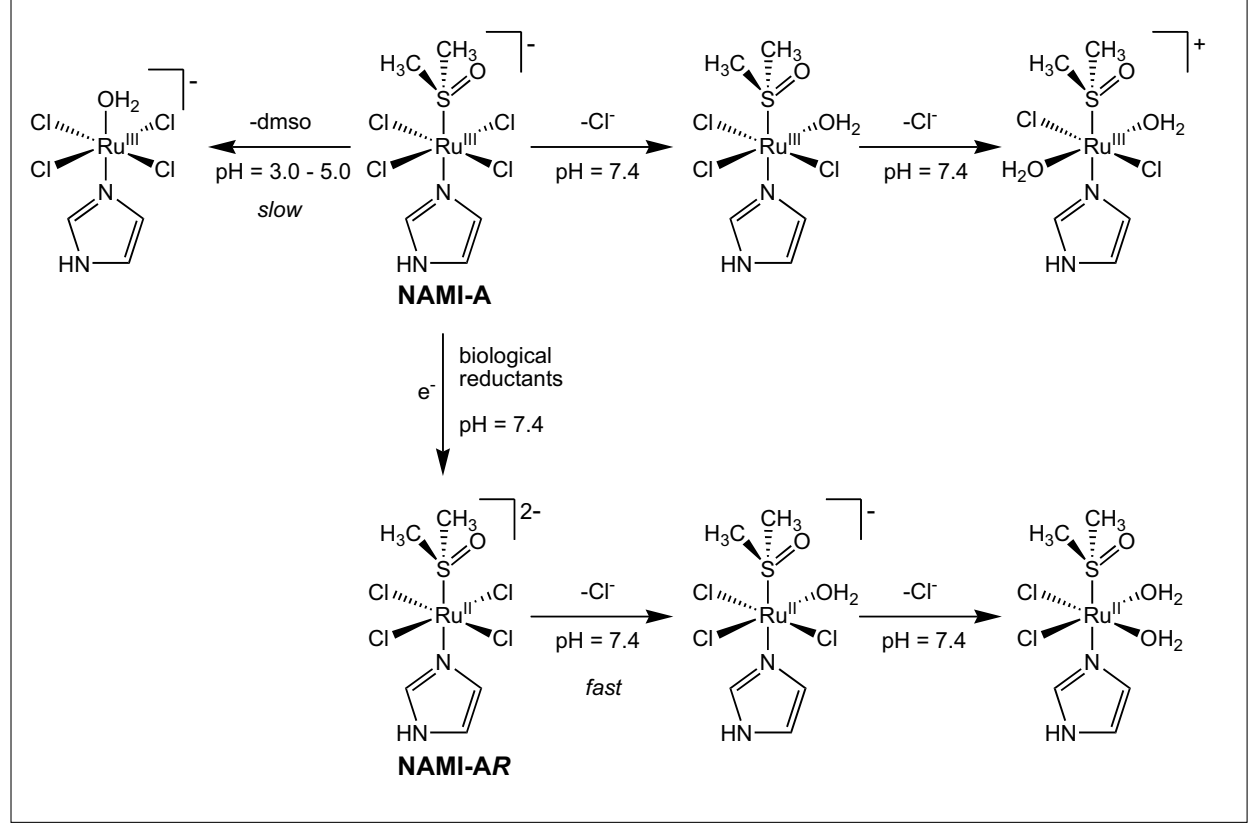

Scheme 1. Schematic representation of the chemical behavior of NAMI-A under various conditions. The geometry of the diaquo species, either cis or trans, is unknown.

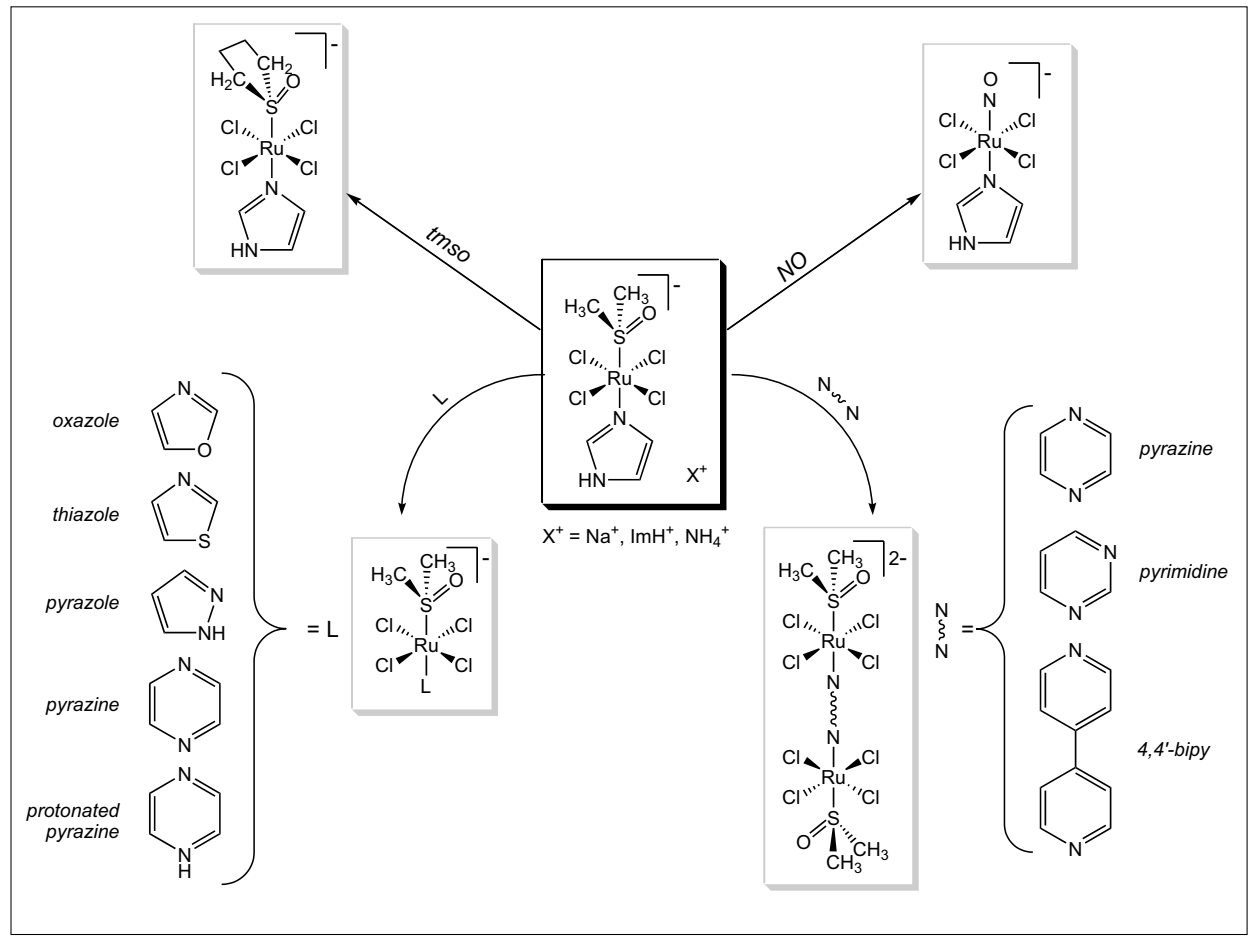

Scheme 2. Schematic representation of the NAMI-A-type compounds

utes by a two-fold excess of glutathione at $37.0^{\circ} \mathrm{C}$ in a $\mathrm{pH}$ range from 4.0 to $7.4 .^{[19]}$ In contrast, Keppler and co-workers ${ }^{[20]}$ established that ICR is reduced by an excess of gluthatione or ascorbic acid only at $\mathrm{pH}$ 7.4 but not, contrary to a previous report, ${ }^{[21]}$ in pure water. Conversely, the redox potential of the indazole derivative KP1019 was found to be remarkably higher than that of $\mathrm{ICR}, c a .0 .00 \mathrm{~V} v s$. NHE, and this complex is completely reduced within minutes by a four-fold excess of ascorbic acid in a $\mathrm{pH} 6$ buffered solution. ${ }^{[20]}$

In conclusion, even though reduction of NAMI-A to Ru(II) is likely to occur in vivo (reducing agents might be glutathione in the cell, where it is present in concentrations ranging from 0.5 to $10 \mathrm{mM}$, or ascorbic acid in the blood, whose concentration range is $11-79 \mu \mathrm{M})$, it is not needed for activation. Owing to its relative lability at physiological $\mathrm{pH}$, activation of NAMI-A may easily occur through aquation. In other words, for NAMI-A the activation-by-reduction hypothesis is a concrete possibility but not a prerequisite to explain its activity. On the other hand, the antimetastatic activity of NAMI-A is most likely strictly related to the presence of dmso in the coordination sphere of its active metabolite(s).

\section{Structure-Activity Relationships}

Over the years, in the attempt to establish structure-activity relationships for NAMI-A, we have systematically investigated each component of the NAMI-A frame. ${ }^{[13]} \mathrm{A}$ short summary of the results is reported here (Scheme 2).

\section{Sulfoxide}

Among the several sulfoxides tested, we found that only tetramethylenesulfoxide (tmso) is well behaved as dmso and leads to the same type of $\mathrm{Ru}$ complexes. The [Na] trans-[RuCl${ }_{4}(\mathrm{Im})($ tmso-S $\left.)\right]$ complex behaved quite similarly to NAMI both from the chemical and the biological point of view. Dmso was also successfully replaced by the stronger $\pi$-acceptor ligand $\mathrm{NO}$, which binds as $\mathrm{NO}^{+}$and leads to the formal reduction of $\mathrm{Ru}(\mathrm{III})$ to $\mathrm{Ru}(\mathrm{II})$. The diamagnetic complex $\left[(\mathrm{Im})_{2} \mathrm{H}\right]$ trans-[ $\left.\mathrm{RuCl}_{4}(\mathrm{Im})(\mathrm{NO})\right]$, which formally derives from NAMI-A by replacement of dmso-S with $\mathrm{NO}^{+}$, is moderately cytotoxic in vitro but is devoid of antimetastatic activity against in vivo models.

\section{N-ligand}

This is the component of the NAMI-A frame that can be more easily varied in a systematic manner. In fact, one of the two trans S-bonded sulfoxides in the $\mathrm{Ru}(\mathrm{III})$ precursor $\left[(\mathrm{dmso})_{2} \mathrm{H}\right]$ trans $-\left[\mathrm{RuCl}_{4}(\mathrm{dmso}\right.$ $\mathrm{S})_{2}$ ] can be easily and selectively replaced, under mild conditions, by a large number of heterocyclic N-ligands (L), and by ammonia as well, affording a series of NAMI-Atype compounds of general formula (HL) trans-[ $\left.\mathrm{RuCl}_{4}(\mathrm{~L})(\mathrm{dmso}-\mathrm{S})\right]$. We found that complexes with large aromatic ligands and increased lipophylicity (e.g. $\mathrm{L}=$ indazole or quinoline), are more toxic but less active than NAMI-A and were not pursued further. More interestingly, NAMI-A-type compounds with $\mathrm{N}$-ligands of comparable size but lower basicity than imidazole (e.g. $\mathrm{L}=$ thiazole) have an in vivo effectiveness slightly better than NAMI-A. In addition, while they behave similarly to NAMI-A at physiological $\mathrm{pH}$, they are more stable than NAMI-A in slightly acidic aqueous solution (i.e. the dissociation of dmso is slower). This feature is beneficial from the point of view of the administration of the drug. By using potentially bridging ligands $(e . g . \mathrm{L}=$ pyrazine, pyrimidine or 4,4'-bipyridine) we also 
prepared dinuclear compounds of formula $[\mathrm{Na}]_{2}\left\{\right.$ trans- $\left.\left[\mathrm{RuCl}_{4}(\mathrm{dmso}-\mathrm{S})\right]_{2}(\mu-\mathrm{L})\right\}$, in which each ruthenium center has a coordination environment similar to that of the NAMI-A-type complexes. We anticipated that dimers might show some new biological features: while the monomeric NAMI-A-type species are either mono- or bifunctional binders after chloride hydrolysis, the new dimers might behave as bi- or even tetra-functional binders. However, in vivo, these compounds show an antimetastatic activity comparable to that of NAMI-A.

\section{Charge}

We established since the early days of NAMI that neutral Ru(III) compound mer$\left[\mathrm{RuCl}_{3}(\mathrm{Im})(\mathrm{dmso}-\mathrm{S})(\mathrm{dmso}-\mathrm{O})\right]$, formally derived from NAMI-A upon replacement of a chloride with a dmso-O, has antimetastatic activity similar to that of NAMI-A. This, and similar neutral compounds, were not pursued further because of their relatively poor solubility in water.

\section{Chlorides}

As described above, the chemical behavior of NAMI-A (and, in general, of all NAMI-A-type complexes) in physiological aqueous solution is dominated by the stepwise hydrolysis of at least two chlorides. Chloride dissociation is also the main chemical process that occurs after reduction of NAMI-A, a process that is likely to occur in vivo. Thus we reasoned that the strategy that in the 80s led from cisplatin to carboplatin might be worth being pursued also for active $\mathrm{Ru}$-chloride-dmso compounds. In fact, replacement in NAMI-A of chlorides with chelating dicarboxylates is expected to induce significant differences in its hydrolytic behavior and, as a consequence, in its activity (it is impossible to anticipate if for better or for worse). We thoroughly investigated the reactivity of NAMI-A and of its precursor $\left[(\mathrm{dmso})_{2} \mathrm{H}\right]$ trans $-\left[\mathrm{RuCl}_{4}\left(\mathrm{dmso}^{-}\right.\right.$ $\mathrm{S})_{2}$ ] towards a number of dicarboxylate ligands (dicarb). Despite our efforts, we were unfortunately unable to isolate any $\mathrm{Ru}(\mathrm{III})$ compound bearing both dmso and a chelating dicarboxylate in its coordination sphere. All the products isolated either did not contain dmso any longer or, more often, were $\mathrm{Ru}$ (II) species. On the other hand, in a systematic investigation we have successfully isolated and characterized several new types of $\mathrm{Ru}(\mathrm{II})$-dmso-dicarb compounds derived from the Ru(II)-dmso precursors 1 and 2. ${ }^{[22]}$

\section{Oxidation State}

As already explained NAMI-A is efficiently reduced by biological reductants. Actually, we found that NAMI-AR, obtained by reduction of NAMI-A with ascorbic acid in vial immediately prior to administration, is even more effective than NAMI-A itself against metastasis growth. ${ }^{[23]}$

\section{Future Challenges and Perspectives}

From the history of anticancer $\mathrm{Ru}$ compounds it clearly emerges that, over the years, basically three classes of active compounds (by active we mean here compounds that have demonstrated effectiveness in vivo against animal models or transplanted human tumors) have been discovered: Ru-dmso compounds developed by our group, $\mathrm{Ru}(\mathrm{III})$ complexes of the type $[\mathrm{LH}]$ trans- $\left[\mathrm{RuCl}_{4}(\mathrm{~L})_{2}\right]$, and organometallic $\mathrm{Ru}(\mathrm{II})$-arene compounds of general formula $\left[\left(\eta^{6}\right.\right.$-arene $\left.) \mathrm{Ru}(\mathrm{en}) \mathrm{Cl}\right]\left[\mathrm{PF}_{6}\right]$. The three groups of active compounds share few common chemical and biological features, aside from the fact that they all contain ruthenium: two are formed by anionic $\mathrm{Ru}$ (III) complexes (and we already pointed out that their structural similarity is indeed deceptive), the other by cationic $\mathrm{Ru}(\mathrm{II})$ organometallic compounds; these latter are quite cytotoxic in vitro, while the others (and NAMI-A in particular) are not. Their in vivo anticancer activity is different: some are active against the primary tumor, others against metastases only. Some compounds are believed to interact with DNA, while for the others this is more controversial or definitely unlikely (e.g. for NAMI-A).

As a consequence, the main challenge for the inorganic chemists involved in this field derives from the absence, at the moment, of any general guideline towards the synthesis of new active species. The discovery of these classes of active compounds was fortuitous. Within each class some structure-activity relationships were found (for example $\mathrm{Sa}$ dler and co-workers showed that, for the organometallic compounds, an increase in the hydrophobicity of the arene leads to increased in vitro cytotoxicity $\left.{ }^{[8]}\right)$, but there are no general rules similar to those established since the early days for Pt drugs.

Therefore, which other Ru compounds might be expected to be active and should be investigated in the future? At the moment this fundamental question has no answer.

Another important challenge, that concerns more the bio-oriented researchers of this field rather than the synthetic inorganic chemists, is that of the screening of new compounds. The classical approach for screening metal-based anticancer compounds, which has so far worked very well for Pt compounds, follows a strategy developed on the classical mechanism of action depicted above. It relies primarily on in vitro models and can be roughly summarized on this sentence of $\mathrm{B}$. Rosemberg, the 'father' of cisplatin: "Not all cellular poisons are cancer drugs, but all cancer drugs are cellular poisons". ${ }^{[1 c]}$ While this strategy is likely to select other classical anticancer drugs, i.e. cytotoxic compounds similar to cisplatin, it is questionable if it may lead to the discovery of alternative, non-classical drugs that might function through different (even though as yet unknown) mechanisms of action. In other words, it is questionable if cytotoxicity tests should be the sole determinants for drug selection, in particular for the screening of new metal-based compounds. We have shown that many active ruthenium complexes have a low general toxicity as compared to established Pt drugs and some of them are not cytotoxic at all in vitro. For example, NAMI-A failed the NCI in vitro evaluation protocol against a panel of 60 cell lines and would have been discarded for further evaluation in the conventional screening if Sava and co-workers had not demonstrated the in vivo antimetastatic potential of this compound. On the other hand, in vivo tests for preliminary screening are highly unpractical, extremely expensive and unethical. Therefore new in vitro protocols have to be found. For example, Sava and co-workers established that, for NAMIA-type compounds, in vitro inhibition of tumor invasion correlates with the in vivo inhibition of metastasis formation. [13] Thus, it appears that this in vitro test, rather than cytotoxic tests, is suitable for determining the antimetastatic efficacy of (at least) this type of ruthenium complexes.

Despite these important challenges, the perspectives in the field of ruthenium anticancer agents are encouraging. The serendipitous discovery that NAMI-A manifests outstanding antimetastatic effects in spite of its very low direct cytotoxicity, i.e. in the absence of cytotoxic effects that involve metal-DNA interactions, indicates that not all anticancer metal complexes act through a cisplatin-like cytotoxic mechanism, and therefore opens the way to the rational design of new 'non-classical' (or targeted) anticancer drugs. A better understanding of the mechanism of action of new drugs such as NAMI-A will be very helpful towards this instance.

Received: July 17, 2007

[1] a) D. Lebwohl, R. Canetta, Eur. J. Canc. 1998, 34, 1522; b) E. Wong, C. M. Giandomenico, Chem. Rev. 1999, 99, 2451; c) 'Cisplatin: Chemistry and Biochemistry of a Leading Anticancer Drugs', Ed. B. Lippert, VHCA, Zürich, Wiley-VCH, Weinheim, 1999.

[2] G. Giaccone, Drugs 2000, 59, 9.

[3] W. H. Ang, P. J. Dyson, Eur. J. Inorg. Chem. 2006, 4003.

[4] P. J. Dyson, G. Sava, Dalton Trans. 2006, 1929.

[5] A. Bergamo, G. Sava, Dalton Trans. 2007, 1267.

[6] a) Z. Guo, P. J. Sadler, Angew. Chem., Int. 
Ed. 1999, 38, 1512; b) M. J. Clarke, Coord. Chem. Rev. 2002, 232, 69; c) C. X. Zhang, S. J. Lippard, Curr. Op. Chem. Biol. 2003, 7, 481; d) L. Ronconi, P. J. Sadler, Coord. Chem. Rev. 2007, 251, 1633.

[7] a) M. J. Clarke, F. Zhu, D. R. Frasca, Chem. Rev. 1999, 99, 2511; b) M. J. Clarke, Coord. Chem. Rev. 2003, 236, 209.

[8] Y. K. Yan, M. Melchart, A. Habtemariam, P. J. Sadler, Chem. Commun. 2005, 4764.

[9] $\mathrm{Ru}(\mathrm{IV})$ compounds are also possible, but require a number of acido, oxo or sulfido ligands for stabilization.

[10] a) G. Mestroni, E. Alessio, G. Sava, S. Pacor, M. Coluccia, in 'Metal Complexes in Cancer Chemotherapy', Ed. B. K. Keppler, VCH: Weinheim (Germany), 1993, p. 157; b) G. Mestroni, E. Alessio, G. Sava, S. Pacor, M. Coluccia, A. Boccarelli, Metal-Based Drugs 1994, 1, 41.

[11] For a recent review of the chemistry of Ru-dmso complexes see E. Alessio, Chem. Rev. 2004, 104, 4203.

[12] B. K. Keppler, K.-G. Lipponer, B. Stenzel, F. Kratzin in 'Metal Complexes in Cancer Chemotherapy', Ed. B. K. Keppler, VCH: Weinheim, Germany, 1993, p. 187.

[13] a) G. Sava, E. Alessio, A. Bergamo, G. Mestroni, in 'Topics in Biological Inorganic Chemistry, Volume 1: Metallopharmaceuticals', Eds. M. J. Clarke, P. J. Sadler, Springer, Berlin, 1999, p. 143; b) E. Alessio, G. Mestroni, A. Bergamo, G. Sava, in 'Metal Ions in Biological Systems, Volume 42: Metal Ions and Their Complexes in Medication and in Cancer Diagnosis and Therapy', Eds. A. Sigel, H. Sigel, M. Dekker, New York, 2004, p. 323 ; c) E. Alessio, G. Mestroni, A. Bergamo, G. Sava, Curr. Topics Med. Chem. 2004, 4, 1525.

[14] J. M. Redemaker-Lakhai, D. van den Bongard, D. Pluim, J. H. Beijnen, J. H. M. Schellens, Clin. Canc. Res. 2004, 10, 3717.

[15] C. G. Hartinger, S. Zorbas-Seifried, M. A. Jakupec, B. Kynast, H. Zorbas, B. K.
Keppler, J. Inorg. Biochem. 2006, 100 891.

[16] a) A. H. Velders, H. Kooijman, A. L. Spek, J. G. Haasnoot, D. De Vos, J. Reedijk, Inorg. Chem. 2000, 39, 2966; b) A. C. G. Hotze, M. Bacac, A. H. Velders, B. A. J. Jansen, H. Kooijman, A. L. Spek, J. G. Haasnoot, J. Reedijk, J. Med. Chem. 2003, 46, 1743.

[17] a) W. H. Ang, E. Daldini, C. Scolaro, R. Scopelliti, L. Juillerat-Jeannerat, P. J. Dyson, Inorg. Chem. 2006, 45, 9006; b) C. A Vock, W. H. Ang, C. Scolaro, A. D. Phillips, L. Lagopoulos, L. Juillerat-Jeanneret, G. Sava, R. Scopelliti, P. J. Dyson, J. Med. Chem. 2007, 50, 2166.

[18] The reduction potential of NAMI-A-type compounds is mainly determined by the presence of dmso-S and the nature of the $\mathrm{N}$-ligand has only marginal influence on its value, see: a) E. Alessio, G. Balducci, A. Lutman, G. Mestroni, M. Calligaris, W.M. Attia, Inorg. Chim. Acta 1993, 203, 205. b) M. Groessl, E. Reisner, C. G. Hartinger, R. Eichinger, O. Semenova, A. R. Timerbaev, M. A. Jakupec, V. B. Arion, B. K. Keppler, J. Med. Chem. 2007, 50, 2185.

[19] M. Ravera, C. Cassino, S. Baracco, D. Osella, Eur. J. Inorg. Chem. 2006, 740.

[20] P. Schluga, C. G. Hartinger, A. Egger, E. Reisner, M. Galanski, M. A. Jakupec, B. K. Keppler, Dalton Trans. 2006, 1796.

[21] M. Hartmann, K.-G. Lipponer, B. K. Keppler, Inorg. Chim. Acta 1998, 267, 137.

[22] a) I. Bratsos, E. Zangrando, B. Serli, N. Katsaros, E. Alessio, Dalton Trans. 2005 , 3881. b) I. Bratsos, B. Serli, E. Zangrando, N. Katsaros, E. Alessio, Inorg. Chem. 2007, 46, 975.

[23] G. Sava, A. Bergamo, S. Zorzet, B. Gava, C. Casarsa, M. Cocchietto, A. Furlani, V. Scarcia, B. Serli, E. Iengo, E. Alessio, G. Mestroni, Eur. J. Cancer 2002, 38, 427. 